\title{
Icariin induces the growth, migration and osteoblastic differentiation of human periodontal ligament fibroblasts by inhibiting Toll-like receptor 4 and NF-kB p65 phosphorylation
}

\author{
HAI-JIANG LIU ${ }^{1}$, XUE-YANG LIU ${ }^{2}$ and DE-BAO JING ${ }^{2}$ \\ ${ }^{1}$ Department of Endodontics, Shanghai Stomatological Hospital, Shanghai 200001; \\ ${ }^{2}$ Department of Stomatology, Gongli Hospital, Shanghai 200135, P.R. China
}

Received December 22, 2017; Accepted June 19, 2018

DOI: $10.3892 / \mathrm{mmr} .2018 .9302$

\begin{abstract}
The proliferation, migration and differentiation capacities of human periodontal ligament fibroblasts (HPDLCs) are important for the treatment of periodontal diseases. The aim of the present study was to investigate whether icariin could promote these abilities in HPDLCs, and explore the cellular mechanisms therein. The results indicated that icarrin markedly blocked apoptosis, and increased the viability and migration of HPDLCs, particularly at the concentrations of 20 and $50 \mu \mathrm{M}$. In addition, icariin significantly promoted HPDLCs to synthesize extracellular matrix, which was reflected by the decreased expression of matrix matalloproteinase-1 and increased expression of tissue inhibitor of metalloproteinase-1. Furthermore, the levels of bone morphogenetic protein 2, collagen I, osteoprotegerin and alkaline phosphatase were markedly elevated by icariin, indicating that icariin was able to promote the osteogenic differentiation capability of HPDLCs. Icariin also inactivated the Toll-like receptor 4 (TLR)-4/nuclear factor (NF)- $\kappa B$ signaling pathway by suppressing the expression levels of TLR-4 and phosphorylated p65, and by blocking p65 nuclear translocation. These results suggested that icarrin increased the survival, migration and osteoblastic differentiation of HPDLCs by inhibiting the TLR-4/NF- $\kappa$ B signaling pathway.
\end{abstract}

Correspondence to: Mrs. Xue-Yang Liu, Department of Stomatology, Gongli Hospital, 219 Miaopu Road, Pudong New Area, Shanghai 200135, P.R. China

E-mail: liuxueyang0824@126.com

Abbreviations: ECM, extracellular matrix; MMP-1, matrix matalloproteinase-1; TIMP-1, tissue inhibitor of metalloproteinase-1; BMP2, bone morphogenetic protein 2; Col I, collagen I; OPG, osteoprotegerin; TLR-4, Toll-like receptor 4; NF- $\kappa \mathrm{B}$, nuclear factor- $\kappa \mathrm{B}$

Key words: growth, migration, osteoblastic differentiation, TLR-4, $\mathrm{NF}-\kappa \mathrm{B}$

\section{Introduction}

Human periodontal ligament, a band of fibrous connective tissue, has shock absorption ability and prevents tooth and alveolar bone injury during chewing (1). As the most abundant cells in human periodontal ligament, human periodontal ligament fibroblasts (HPDLCs) are involved in pathological changes of the human periodontal ligament and periodontal tissue regeneration (2). Besides that, HPDLCs have been demonstrated to have the capacity to generate extracellular matrix and regulate osteoclastic differentiation within periodontal tissue (3). For these reasons, HPDLCs was considered to play a vital role in the damage repair of periodontal diseases.

Icariin (2-(4'-methoxyphenyl)-3-rhamnosido-5-hydroxyl7-glucosido-8-(3'-methyl-2-butylenyl)-4-chromanone, the major active ingredient of epimedii, possesses a broad spectrum of pharmacological applications, such as the protection of bone, cartilage, neuro and cardiovascular system (4). In addition, it has been suggested and supported by studies that icarrin could induce rat adipose-derived stem cells differentiation into osteoblasts (5) and regulate extracellular matrix (ECM) synthesis (6). However, little is known that whether icariin could affect ECM synthesis and osteoblastic differentiation in HPDLCs.

Matrix metalloproteinases (MMPs) are a family of proteolytic enzymes that degrade components of ECM (7). According to published reports, numerous pathways such as Toll-like receptors (TLRs) (8), NF- $\kappa \mathrm{B}$ (9) play important roles in MMPs expressions. However, activation of NF- $\kappa \mathrm{B}$ and TLRs inhibit osteogenic differentiation in various cells, including pre-osteoblasts (10), human bone marrow mesenchymal stem cells (11). Therefore, TLR/NF- $\kappa$ B pathway maybe a major target for the prevention and treatment of damaged HPDLCsinduced periodontal diseases.

In the present study, HPDLCs were isolated and cultured in the absence or presence of icariin at different concentrations to explore the molecular mechanisms of icarrin in HPDLCs. These results strongly demonstrated that icariin promote HPDLCs to differentiate into osteoblasts and stimulate ECM synthesis via suppressing TLR-4 and phosphorylation of $\mathrm{NF}-\kappa \mathrm{B}$. 


\section{Materials and methods}

Primary culture of HPDLCs. HPDLCs were isolated from the molar of a female patient (25 years old) at Shanghai Stomatological Hospital. Briefly, periodontal ligament tissue dissected from patients were chopped into pieces and digested with $0.25 \%$ Trypsin/EDTA (Gibco; Thermo Fisher Scientific, Inc., Waltham, MA, USA) at $37^{\circ} \mathrm{C}$ for $1 \mathrm{~h}$. Then, cells were centrifugated at $1,000 \mathrm{x}$ g for $10 \mathrm{~min}$ and subsequently cultured in Dulbecco's modified Eagle's medium (DMEM) (Gibco; Thermo Fisher Scientific, Inc.) containing 10\% fetal bovine serum (FBS) at $37^{\circ} \mathrm{C}$ with $5 \% \mathrm{CO}_{2}$. A complete clinical examination was performed in all participants, clinical data were recorded and written consent was obtained from each subject. The experimental protocols were approved by the Ethics Committee of Shanghai Stomatological Hospital (Shanghai, China).

CCK-8 assay. Icariin was obtained from the Chinese National Institute for Control of Pharmaceutical and Biological Products (Beijing, China). Cell viability was measured using Cell Counting Kit-8 (CCK-8) (Dojindo Molecular Technologies, Inc., Kumamoto, Japan ). Briefly, HPDLCs were planted into a 96 -well plate at the concentration of $5 \times 10^{3} /$ well. After adherence, cells were treated with icariin at different concentrations for different times. Then, CCK- 8 solution was added into cells, and cells were incubated for another $4 \mathrm{~h}$. The average value of optic density was detected using a Microplate Spectrophotometer (Thermo Fisher Scientific, Inc.) at a wavelength of $450 \mathrm{~nm}$.

Detection of cell apoptosis. HPDLCs were cultured in the in the absence and presence of various concentrations of icariin for $24 \mathrm{~h}$. Then, cells packed by centrifugation at $1,000 \mathrm{x} \mathrm{g}$. After washing with PBS for 3 times, cells were stained with Hoechst 33258 at $37^{\circ} \mathrm{C}$ for 5 min in the dark. Images (magnificaion, $\mathrm{x} 400$ ) were captured under a confocal laser scanning microscope (Carl Zeiss AG, Oberkochen, Germany).

Transwell assay. $2 \times 10^{4}$ cells in each group were seeded into serum-free medium in the insert coated with Matrigel (BD Biosciences, San Jose, CA, USA), and the lower chamber was filled with DMEM containing 10\% FBS. After incubation for $24 \mathrm{~h}$, the invaded cells were stained with crystal violet. Cells were counted in at least 6 randomly selected fields under a light microscope (Olympus, Tokyo, Japan).

Western blot analysis. Proteins were isolated from HPDLCs using a Total Protein Extraction kit (Applygen Technologies, Inc., Beijing, China). Then, proteins were separated by $10 \%$ sodium dodecyl sulfate-polyacrylamide gel electrophoresis (SDS-PAGE) and transferred onto polyvinylidene difluoride (PVDF) membranes (EMD Millipore, Billerica, MA, USA). After blocking with 5\% BSA, membranes were incubated with primary antibodies specific for matrix matalloproteinase-1 (MMP-1), bone morphogenetic protein 2 (BMP2), collagen I (Col I), osteoprotegerin (OPG), toll-like receptor 4 (TLR-4), nuclear factor- $\kappa \mathrm{B}(\mathrm{NF}-\kappa \mathrm{B})$, GAPDH (Abcam, Cambridge, MA, USA) at $4^{\circ} \mathrm{C}$ overnight, followed by incubation with horseradish peroxidase-conjugated $\operatorname{IgG}$ at room temperature for $1.5 \mathrm{~h}$. Blots were observed using enhanced chemiluminescence.

ELISA assay. HPDLCs were planted into a 96-well plate at the concentration of $2 \times 10^{5}$ cells $/ \mathrm{ml}$. After different treatment, the cells were incubated for another $24 \mathrm{~h}$. Then, culture supernatants were collected and ALP level was assessed using ELISA kits (R\&D Systems, Inc., Minneapolis, MN, USA). The experiment performed strictly according to the manufacturer's instructions.

Detection of p65 nuclear translocation. HPDLCs were cultured in chamber slides, followed by different treatment. Then, cells were fixed in $4 \%$ paraformaldehyde, permeabilized with $0.1 \%$ Trion and blocked with 5\% BSA in PBS for $1 \mathrm{~h}$ at room temperature. Then, cells were incubated with NF- $\mathrm{kB}$ p65 (Abcam) at $4^{\circ} \mathrm{C}$ overnight, followed by incubation with second antibody for $30 \mathrm{~min}$. After washing, cells were counterstained with DAPI. Cells were observed under a confocal laser scanning microscope (Carl Zeiss Group, Germany).

Flow cytometry. Cell apoptosis was measured using Annexin V Apoptosis Detection kit (BD Biosciences). Briefly, HPDLCs were treated with Icariin at different concentrations for $24 \mathrm{~h}$. Then, cells were stained with Annexin V-FITC and PI for $15 \mathrm{~min}$. Cell apoptosis was detected using FACS Calibur flow cytometer (BD Biosciences).

Statistical analysis. All statistical analysis was carried out using SPSS 17.0 (SPSS, Inc., Chicago, IL, USA). Data are presented as the mean \pm standard deviation. Analysis was performed using one-way analysis of variance followed by a Bonferroni post hoc test. $\mathrm{P}<0.05$ was considered to indicate a statistically significant difference.

\section{Results}

The effect of icariin on HPDLCs viability. The structure of icariin was shown in Fig. 1A. To select the proper concentration of icariin for subsequent experiments, HPDLCs were treated with icariin at different concentration $(0.1,0.25,0.5$, $1,2.5,5,10,20,50,100,200,300$ and $400 \mu \mathrm{M}$ ) for $24 \mathrm{~h}$. As illustrated in Fig. 1B, cell viability was remarkably suppressed in HPDLCs cells treated with icariin at the concentration of $50 \mu \mathrm{M}$ above. To exclude cell toxicity, the concentrations of 10,20 and $50 \mu \mathrm{M}$ were chosen for the next experiments. CCK-8 assay results indicated that cell viability was increased in a dose-dependent manner when treated by icariin at day 2, 3 and 4 (Fig. 1C).

Icariin suppresses the apoptosis of HPDLCs. To investigate whether icariin was related to cell apoptosis, the morphological changes of icariin-treated HPDLCs were measured by Hoechst 33258 staining and flow cytometry. Results suggested that the icariin treatment decreased the cell apoptosis in a dose-dependent manner (Fig. 2).

Icariin induces HPDLCs motility and fibrosis. To explore the effect of icariin on the motility of HPDLCs, cell motility was measured using Transwell. As illustrated in Fig. 3A, cell 
<smiles>COc1ccc(-c2oc3c(CC=C(C)C)c(O[C@@H]4O[C@H](CO)[C@@H](O)[C@H](O)[C@H]4O)cc(O)c3c(=O)c2O[C@@H]2O[C@H](C)[C@@H](O)[C@H](O)[C@H]2O)cc1</smiles>

C33H40015 MW : 676.66
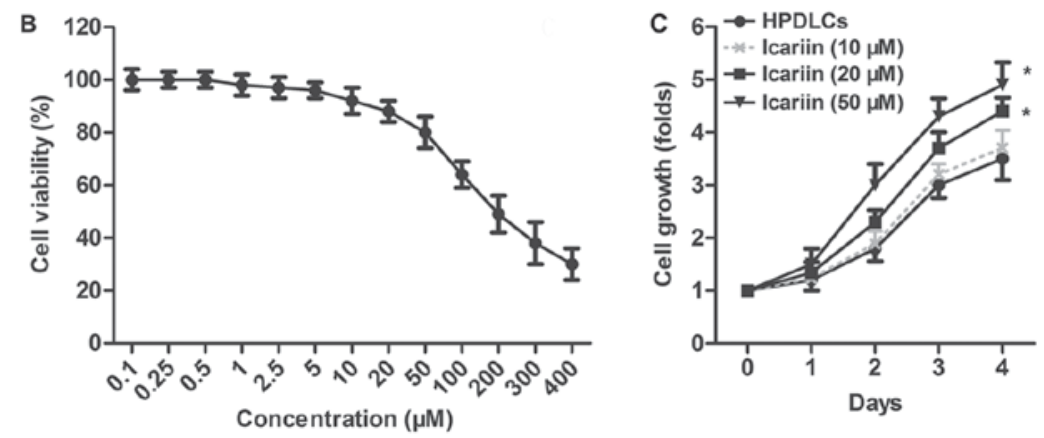

Figure 1. Effect of icariin on HPDLC proliferation. (A) The structure of icariin. (B) HPDLC viability was detected by CCK-8 assay. Cells were treated with icariin at different concentrations $(0.1,0.25,0.5,1,2.5,5,10,20,50,100,200,300$ and $400 \mu \mathrm{M})$ for $24 \mathrm{~h}$. (C) Cell viability was measured using CCK-8 in HPDLC cells treated with icariin at different concentrations $(0,10,20$ and $50 \mu \mathrm{M})$ and different times $(1,2,3$ and 4 days). The experiments were repeated at least 3 times with similar results, and data are presented as the mean \pm standard deviation. ${ }^{*} \mathrm{P}<0.05$ vs. HPDLC. CCK, Cell Counting Kit; HPDLC, human periodontal ligament fibroblast.

A
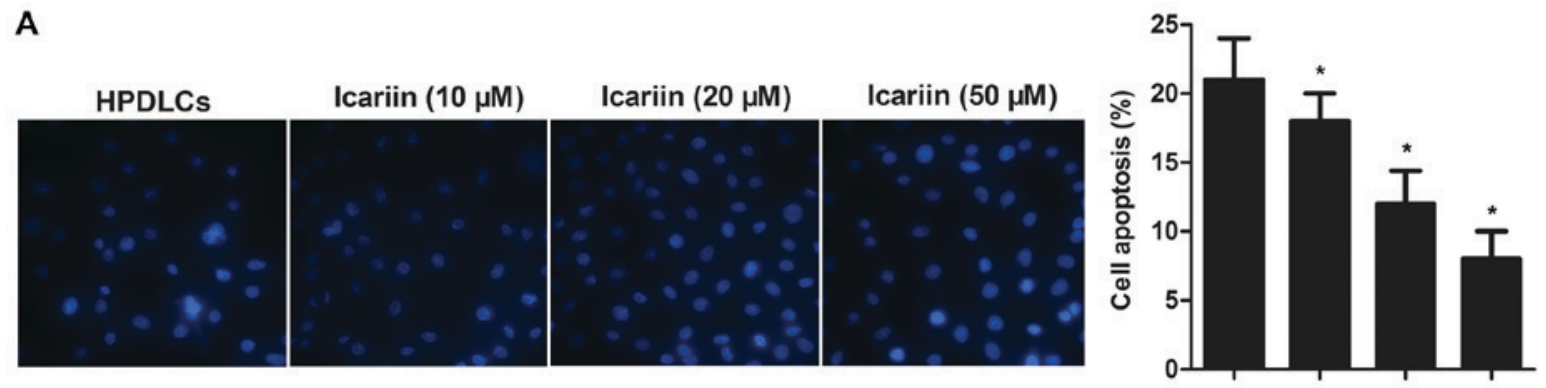

B
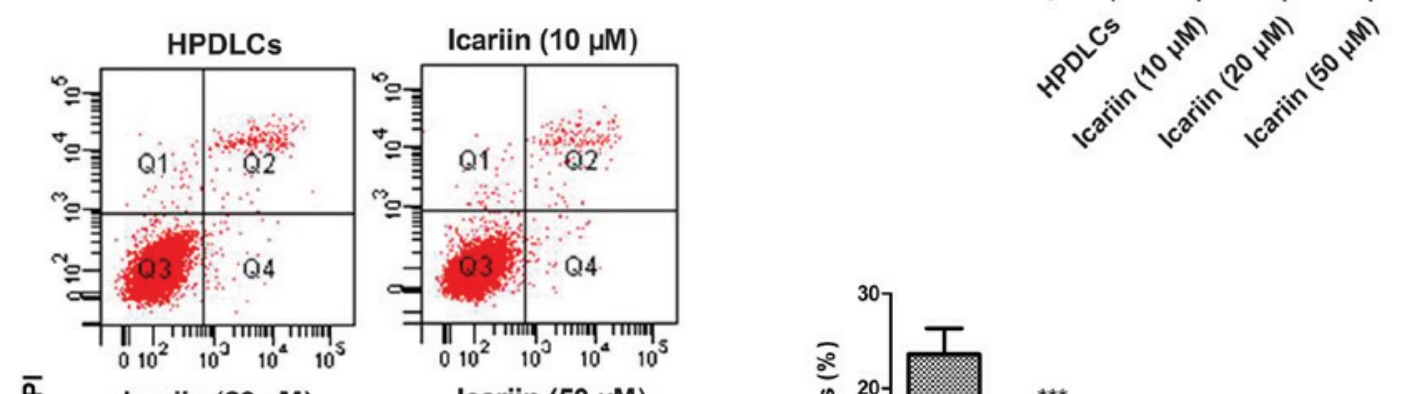

$\overline{\mathbf{x}}$ Icariin $(50 \mu \mathrm{M})$
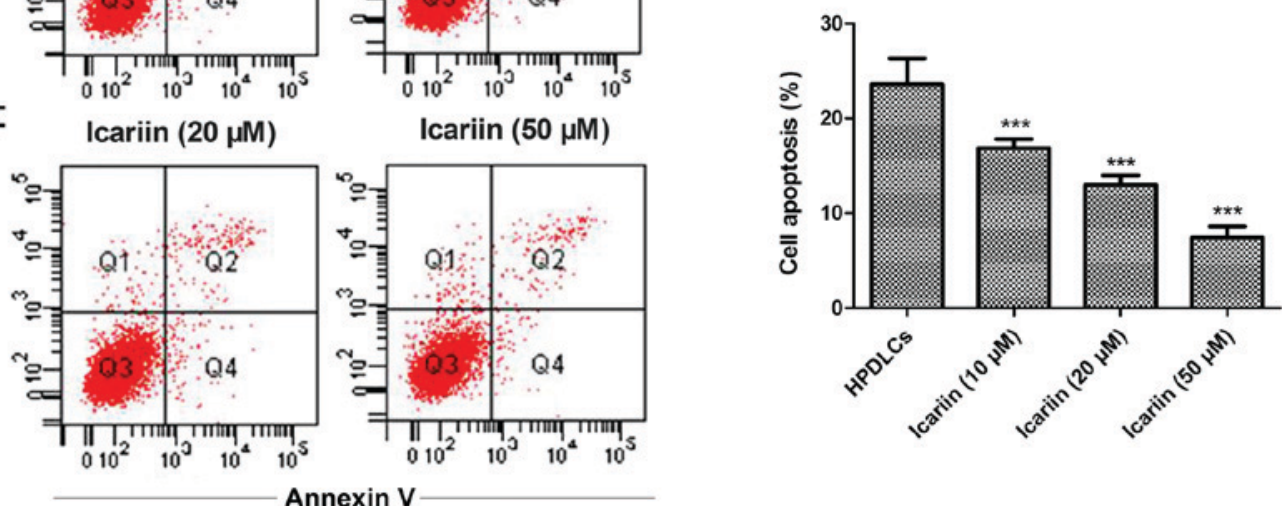

Figure 2. Icariin suppresses the apoptosis of HPDLCs. HPDLCs were administered icariin at different concentrations (0, 10, 20 and $50 \mu \mathrm{M})$ for 24 h. (A) Cell apoptosis was determined using immunofluorescence assay (magnification, x400). (B) Histogram representing the statistical analysis of the rate of cell apoptosis. The experiments were repeated at least 3 times with similar results, and data are presented as the mean \pm standard deviation. ${ }^{*} \mathrm{P}<0.05$ and ${ }^{* * *} \mathrm{P}<0.001$ vs. HPDLCs. HPDLC, human periodontal ligament fibroblast; PI, propidium iodide.

motility increased in a dose-dependent manner when treated with icariin. Besides that, MMP-1 expression was reduced and TIMP-1 expression was elevated with the increasing concentration of icariin (Fig. 3B), indicating that icariin play a vital role in inducing HPDLCs fibrosis.

Icariin enhances HPDLCs osteogenic differentiation ability. To determine whether icariin could affect the osteogenic differentiation of HPDLCs, the level of related proteins were measured using western blotting. Results suggested that, BMP2, Col I and OPG expressions were slightly elevated in icariin $(20 \mu \mathrm{M})$ group and remarkably increased in icariin $(50 \mu \mathrm{M})$ group compared with control group (Fig. 4A). In addition, ELISA assay indicated that icariin treatment enhanced ALP level in a dose-dependent manner in the supernatant of HPDLCs (Fig. 4B). These results indicated that HPDLCs 

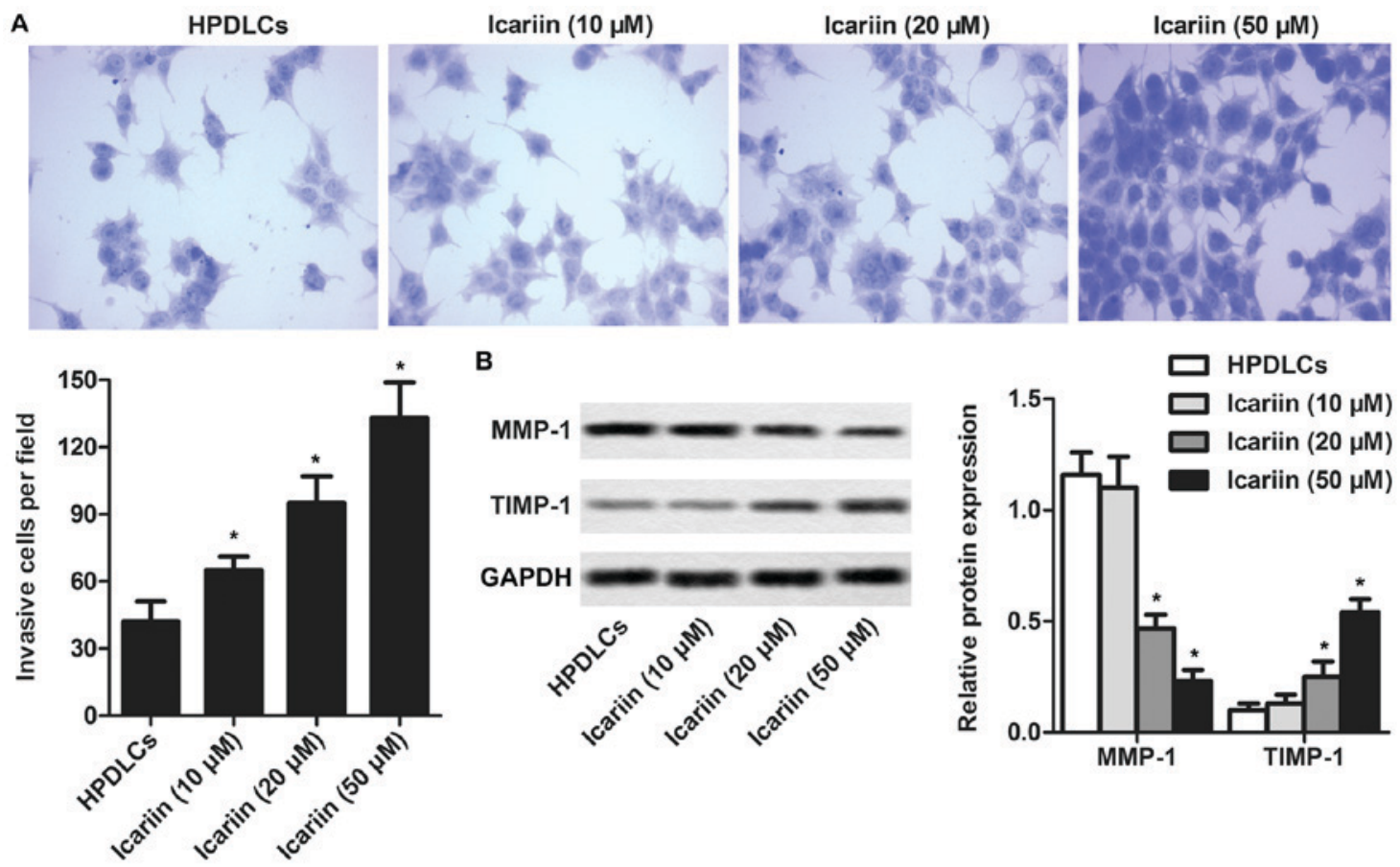

Figure 3. Icariin induces HPDLC motility and fibrosis. HPDLCs were administered icariin at different concentrations $(0,10,20$ and $50 \mu \mathrm{M})$ for $24 \mathrm{~h}$. (A) A Transwell assay was performed to assess the migration ability of HPDLCs (magnification, x400). (B) The expression levels of MMP-1 and TIMP-1 were evaluated by western blotting. The experiments were repeated at least 3 times with similar results, and data are presented as the mean \pm standard deviation. ${ }^{*} \mathrm{P}<0.05$ vs. HPDLCs. HPDLC, human periodontal ligament fibroblast; MMP-1, matrix matalloproteinase-1; TIMP-1, tissue inhibitor of metalloproteinase-1.

A

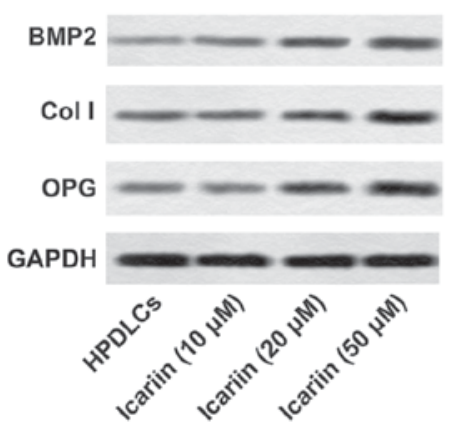

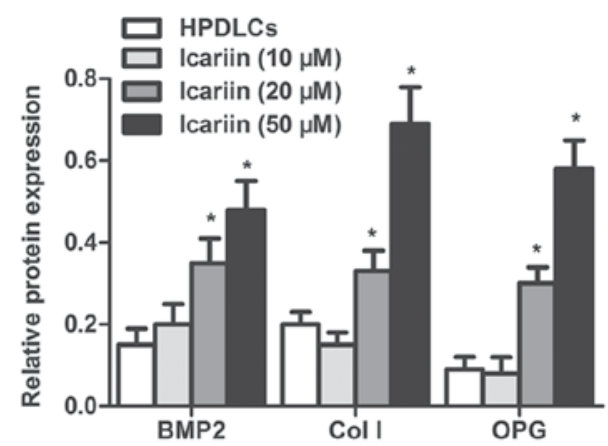

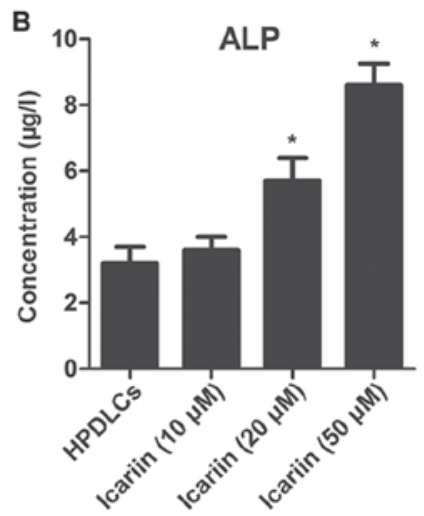

Figure 4. Icariin enhances HPDLC osteogenic differentiation ability. HPDLCs were administered icariin at different concentrations $(0,10,20$ and $50 \mu \mathrm{M})$ for $24 \mathrm{~h}$. (A) The levels of BMP2, Col I and OPG were detected by western blotting. (B) ELISA was employed to evaluate the ALP levels. The experiments were repeated at least 3 times with similar results, and data are presented as the mean \pm standard deviation. ${ }^{*} \mathrm{P}<0.05$ vs. HPDLCs. HPDLC, human periodontal ligament fibroblast; BMP2, bone morphogenetic protein 2; Col I, collagen I; OPG, osteoprotegerin; ALP, alkaline phosphatase.

osteogenic differentiation ability could be enhanced by icariin treatment.

Icariin inactivates $T L R-4 / N F-\kappa B$ pathway. Recently, various researches have suggested that the activation of TLR-4/NF- $\mathrm{B}$ pathway could inhibit osteogenic differentiation, cell viability and motility. Thus, TLR-4/NF- $\mathrm{B}$ pathway was examined. It is observed that icarrin decreased TLR-4 expression and p65 phosphorylation in a dose-dependent manner (Fig. 5A). Moreover, p65 nuclear translocation was remarkably suppressed by icariin treatment (Fig. 5B). These results indicated that TLR-4/NF- $\kappa$ B pathway could be remarkably suppressed by icariin treatment.

\section{Discussion}

HPDLCs is one of the most important cells in periodontal ligament. Due to the stem cell-like property, HPDLCs have potent proliferation, differentiation and migration abilities (12). According to published reports, HPDLCs could differentiate into osteoblastic cells and collagen-forming cells (13). Accumulated studies have indicated that HPDLCs play a vital role in maintaining the integrity and homeostasis of the periodontal ligament during alveolar bone remodeling (14). Thus, improving the proliferation, differentiation and migration capacity is one of the beneficial strategies to suppress periodontal ligament degradation. 

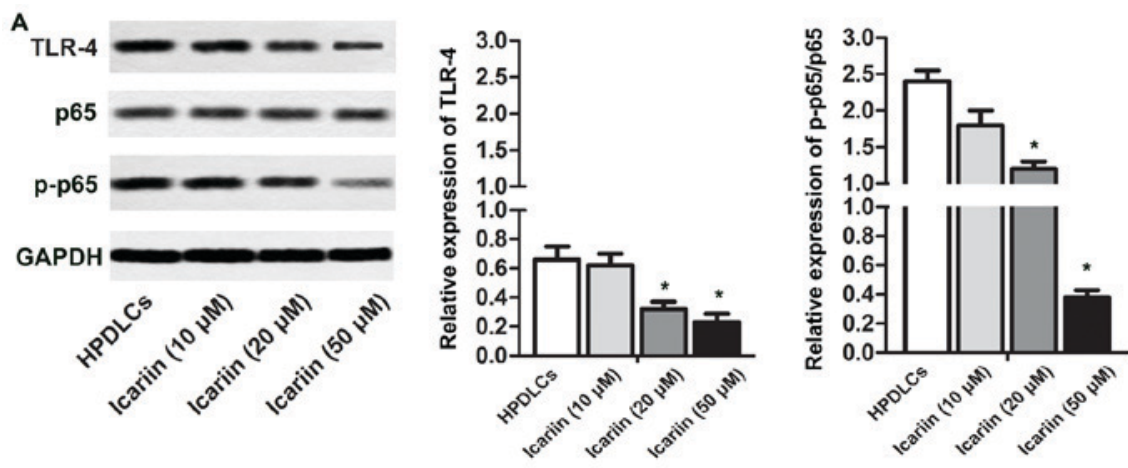

B
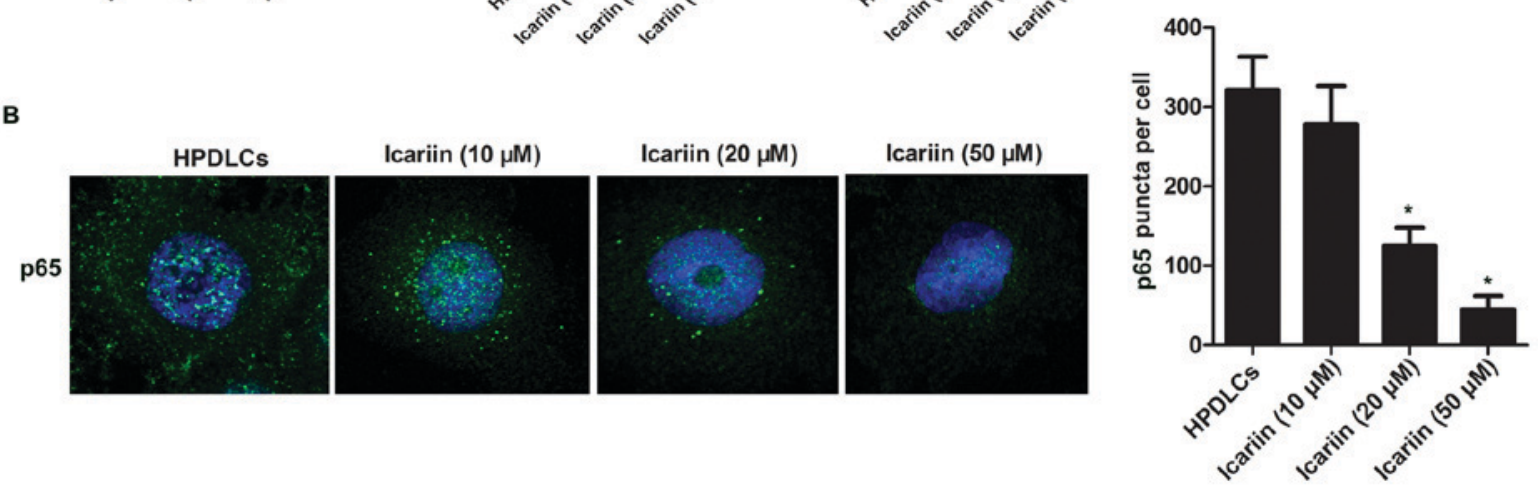

Figure 5. Icariin inactivates the TLR-4/NF-kB signaling pathway. HPDLCs were administered icariin at different concentrations $(0,10,20$ and $50 \mu \mathrm{M})$ for $24 \mathrm{~h}$. (A) Western blot analysis was performed to detect the expression of TLR-4, p65 and p-p65. (B) The nuclear translocation of p65 was observed using an immunofluorescence assay (magnification, $\mathrm{x} 400$ ). The experiments were repeated at least 3 times with similar results, and data are presented as the mean \pm standard deviation. "P<0.05 vs. HPDLCs. HPDLC, human periodontal ligament fibroblast; TLR-4, Toll-like receptor 4; NF- $\mathrm{B}$, nuclear factor- $\mathrm{kB}$; p-, phosphorylated.

Both proliferation and migration of HPDLCs are essential for repair and regeneration. The results from current studies have demonstrated that icariin could promote the proliferation and migration of various cells. For example, icariin was found to tremendously enhance the osteoblast proliferation and Col I level using membrane chromatography coupled with liquid chromatography and time-of-flight mass spectrometry (15). Icariin treatment increased the proliferation of human neural stem cells and the formation of neurospheres (16). Moreover, human umbilical cord mesenchymal stem cells treated with icariin $(100 \mu \mathrm{g} / \mathrm{ml})$ exhibit remarkable cell migration to kidney tissue in mice with acute kidney (17). Endothelial cell migration could be promoted by icariin treatment (18). Similarly, in our research, the proliferation and migration of HPDLCs were tremendously elevated when the concentration of icariin was 20 and $50 \mu \mathrm{M}$.

Previous studies have demonstrated that ECM degradation is closely related to MMPs and TIMPs, for TIMPs help to regenerate ECM via binding to MMPs to decrease their activity (19). Icariin was reported to be involved in the expression of MMPs and TIMPs. According to Wang et al (20), icariin suppressed the MMP-1, MMP-3 and MMP-13 expression via MAPK pathways in IL-1 $\beta$-induced SW1353 chondrosarcoma cells. Icariin treatment inhibits MMP-9 expression and carbonic anhydrase II in the tibia of glucocorticoid-induced hypocalcemia and hypercalciuria mice (21). Lipopolysaccharide-induced the increase in the level of MMP-1, MMP-3, MMP-13, cyclooxygenase- 2 and iNOS could be remarkably suppressed by the pretreatment of icariin in neonatal mice chondrocytes (22). Among the MMPs, MMP-1 is the major enzyme to participate in degrading collagen types I and III, which are the most abundant components of the periodontal tissue matrix (23). In our research, icariin treatment decreased MMP-1 expression and elevated TIMP-1 expression in a dose-dependent manner, indicating ECM degradation could be restrained by icariin in HPDLCs. According to published stidies, MMP-1 expression is always associated with enhanced cell mobility, which is not consistent to what they found. Thus, we speculated that icariin may be involved in some other mechanism that has a moderation effect on MMP-1 expression and cell mobility, which need further exploration.

Apoptosis, a common cellular behavior, has irreplaceable functions in multicellular organisms. Accumulated studies have indicated that HPDLCs apoptosis is closely associated with the development of periodontitis (24). Icariin was reported to possess the anti-apoptosis ability. According to published researches, $\mathrm{H}_{2} \mathrm{O}_{2}$-induced apoptosis was inhibited by icariin via the PI3K/Akt Pathway in rat nucleus pulposus intervertebral disc cells (25). Besides that, oral administration of icariin remarkably suppressed cardiomyocyte apoptosis and attenuated left heart ventricle remodeling and abnormal mitochondria (26). Similarly, our research indicated that the apoptosis of HPDLCs was reduced by icariin in a dose-dependent manner.

BMP2, Col I, OPG and ALP have potent capacity of promoting osteoblast differentiation and inducing osteogenesis $(27,28)$. Arising studies have demonstrated that icarrin play a vital role in regulating these genes. Wang et al used ALP activity assay to observe that icariin stimulate BMP2 osteogenesis in a concentration-dependent manner (29). Besides, the expression of osteogenic genes (Col I, Runx2, osteopotin and DLX5) were significantly elevated by icariin in 
rat bone marrow stromal cells (30). Moreover, Mok et al (31) demonstrated that icariin inhibited the loss of bone mass and strength in the distal pat of the femur and elevated the mRNA level of OPG in the ovariectomized mouse. A similar result was drawn in our research, the expression levels of BMP2, Col I, OPG and ALP were tremendously enhanced by icariin at a dose-dependent manner.

It has been suggested and supported by studies that the inactivation of TLR-4 and NF- $\kappa \mathrm{B}$ was involved in ECM synthesis and osteogenic differentiation $(11,32)$. The results obtained from the current studies have demonstrated that icarrin was closely related to $T L R-4 / N F-\kappa B$ pathway. According to Zhang et al, icariin treatment could remarkably suppress NF- $\kappa \mathrm{B}$ nuclear translocation and the activation of Nlrp3 inflammasome in IgAN rats (33). In addition, icariin can elevate the osteogenic differentiation activity, decrease the $\mathrm{NF}-\kappa \mathrm{B}$ gene and protein expression, increase the OPG expression, enhance of ALP gene expression level in MC3T3-E1 cells (34). A similar result was drawn in our research, the expression of TLR-4 and P-65 was dramatically blocked by icarrin treatment. Moreover, icarrin further suppressed $N F-\kappa B$ p65 nuclear translocation in HPDLCs.

In conclusion, the current study has illustrated that icarrin treatment decreased the apoptosis and increased the viability and migration of HPDLCs. However, HPDLCs have stem cell-like characteristics and the osteogenic differentiation and ECM synthesis abilities could be remarkably enhanced by icariin treatment via inactivating of TLR-4/NF- $\kappa \mathrm{B}$ pathway. Our study is valuable for unraveling the underlying mechanism of icarrin as a candidate drug for periodontal diseases.

\section{Acknowledgements}

The authors would like to thank Shanghai Stomatological Hospital (Shanghai, China) for providing advice and technical support during the present study.

\section{Funding}

No funding was received.

\section{Availability of data and materials}

The datasets used and/or analyzed during the current study are available from the corresponding author on reasonable request.

\section{Authors' contributions}

HJL analyzed and interpreted the main data regarding the cell function study and immunofluorescence. XYL was responsible for study design and the drafting of the manuscript. DBJ conducted the statistical analysis. All authors read and approved the final manuscript.

\section{Ethics approval and consent to participate}

The experimental protocols were approved by the Institute Research Medical Ethics Committee of Shanghai Stomatological Hospital (Shanghai, China); written informed consent was obtained from all participants.

\section{Patient consent for publication}

Written informed consent was obtained from all participants.

\section{Competing interests}

The authors declare that they have no competing interests.

\section{References}

1. Naveh GR, Lev-Tov Chattah N, Zaslansky P, Shahar R and Weiner S: Tooth-PDL-bone cmplex: Response to compressive loads encountered during mastication-a review. Arch Oral Biol 57: 1575-1584, 2012.

2. Roberts WE, Mozsary PG and Klingler E: Nuclear size as a cell-kinetic marker for osteoblast differentiation. Am J Anat 165: 373-384, 1982.

3. Konstantonis D, Papadopoulou A, Makou M, Eliades T, Basdra EK and Kletsas D: Senescent human periodontal ligament fibroblasts after replicative exhaustion or ionizing radiation have a decreased capacity towards osteoblastic differentiation. Biogerontology 14: 741-751, 2013.

4. Li C, Li Q, Mei Q and Lu T: Pharmacological effects and pharmacokinetic properties of icariin, the major bioactive component in Herba Epimedii. Life Sci 126: 57-68, 2015.

5. Ye Y, Jing X, Li N, Wu Y, Li B and Xu T: Icariin promotes proliferation and osteogenic differentiation of rat adipose-derived stem cells by activating the RhoA-TAZ signaling pathway. Biomed Pharmacother 88: 384-394, 2017.

6. Li M, Zhang C, Zhong Y and Zhao J: A novel approach to utilize icariin as icariin-derived ecm on small intestinal submucosa scaffold for bone repair. Ann Biomed Eng 45: 2673-2682, 2017.

7. Parks WC, Wilson CL and López-Boado YS: Matrix metalloproteinases as modulators of inflammation and innate immunity. Nat Rev Immunol 4: 617-629, 2004.

8. Medzhitov R, Shevach EM, Trinchieri G, Mellor AL, Munn DH, Gordon S, Libby P, Hansson GK, Shortman K, Dong C, et al: Highlights of 10 years of immunology in nature reviews immunology. Nat Rev Immunol 11: 693-702, 2011.

9. Shih YL, Chou HM, Chou HC, Lu HF, Chu YL, Shang HS and Chung JG: Casticin impairs cell migration and invasion of mouse melanoma B16F10 cells via PI3K/AKT and NF- $\kappa \mathrm{B}$ signaling pathways. Environ Toxicol 32: 2097-2112, 2017.

10. Wang LM, Zhao N, Zhang J, Sun QF, Yang CZ and Yang PS: Tumor necrosis factor-alpha inhibits osteogenic differentiation of pre-osteoblasts by downregulation of EphB4 signaling via activated nuclear factor $-\kappa \mathrm{B}$ signaling pathway. J Periodontal Res 53: 66-72, 2018.

11. Wang YJ, Zhang HQ, Han HL, Zou YY, Gao QL and Yang GT: Taxifolin enhances osteogenic differentiation of human bone marrow mesenchymal stem cells partially via NF- $\kappa \mathrm{B}$ pathway. Biochem Biophys Res Commun 490: 36-43, 2017.

12. Lim SS, Kook SH and Lee JC: COMP-Ang1 enhances DNA synthesis and cell cycle progression in human periodontal ligament cells via Tie2-mediated phosphorylation of PI3K/Akt and MAPKs. Mol Cell Biochem 416: 157-168, 2016.

13. Kook SH, Jeon YM, Park SS and Lee JC: Periodontal fibroblasts modulate proliferation and osteogenic differentiation of embryonic stem cells through production of fibroblast growth factors, J Periodontol 85: 645-654, 2014.

14. Lekic P and McCulloch CA: Periodontal ligament cell population: The central role of fibroblasts in creating a unique tissue. Anat Rec 245: 327-341, 1996.

15. Wang N, Zhang Q, Xin H, Shou D and Qin L: Osteoblast cell membrane chromatography coupled with liquid chromatography and time-of-flight mass spectrometry for screening specific active components from traditional Chinese medicines. J Sep Sci 40: 4311-4319, 2017.

16. Yang P, Guan YQ, Li YL, Zhang L, Zhang L and Li L: Icariin promotes cell proliferation and regulates gene expression in human neural stem cells in vitro. Mol Med Rep 14: 1316-1322, 2016.

17. Cui H, Liu Z, Wang L, Bian Y, Li W, Zhou H, Chu X and Zhao Q: Icariin-treated human umbilical cord mesenchymal stem cells decrease chronic liver injury in mice. Cytotechnology 69: 19-29, 2017. 
18. Chung BH, Kim JD, Kim CK, Kim JH, Won MH, Lee HS, Dong MS, Ha KS, Kwon YG and Kim YM: Icariin stimulates angiogenesis by activating the MEK/ERK- and PI3K/Akt/eNOSdependent signal pathways in human endothelial cells. Biochem Biophys Res Commun 376: 404-408, 2008.

19. Belibasakis GN and Guggenheim B: Induction of prostaglandin $\mathrm{E}(2)$ and interleukin-6 in gingival fibroblasts by oral biofilms. FEMS Immunol Med Microbiol 63: 381-386, 2011.

20. Wang Z, Ding L, Zhang S, Jiang T, Yang Y and Li R: Effects of icariin on the regulation of the OPG-RANKL-RANK system are mediated through the MAPK pathways in IL-1 $\beta$-stimulated human SW1353 chondrosarcoma cells. Int J Mol Med 34: 1720-1726, 2014

21. Zhang J, Song J and Shao J: Icariin attenuates glucocorticoidinduced bone deteriorations, hypocalcemia and hypercalciuria in mice. Int J Clin Exp Med 8: 7306-7314, 2015.

22. Liu MH, Sun JS, Tsai SW, Sheu SY and Chen MH: Icariin protects murine chondrocytes from lipopolysaccharide-induced inflammatory responses and extracellular matrix degradation, Nutr Res 30: 57-65, 2010.

23. Hannas AR, Pereira JC, Granjeiro JM and Tjäderhane L: The role of matrix metalloproteinases in the oral environment. Acta Odontol Scand 65: 1-13, 2007.

24. Liu J, Jiang Y, Mao J, Gu B, Liu H and Fang B: High levels of glucose induces a dose-dependent apoptosis in human periodontal ligament fibroblasts by activating caspase-3 signaling pathway. Appl Biochem Biotechnol 170: 1458-1471, 2013.

25. Deng $X$, Chen $S$, Zheng $D$, Shao Z, Liang $H$ and Hu H: Icariin prevents $\mathrm{H}_{2} \mathrm{O}_{2}$-induced apoptosis via the PI3K/Akt pathway in rat nucleus pulposus intervertebral disc cells. Evid Based Complement Alternat Med 2017: 2694261, 2017.

26. Qian ZQ, Wang YW, Li YL, Li YQ, Ling-Zhu and Yang DL: Icariin prevents hypertension-induced cardiomyocyte apoptosis through the mitochondrial apoptotic pathway. Biomed Pharmacother 88: 823-831, 2017.

27. Di Benedetto A, Posa F, Carbone C, Cantore S, Brunetti G, Centonze M, Grano M, Lo Muzio L, Cavalcanti-Adam EA and Mori G: NURR1 downregulation favors osteoblastic differentiation of MSCs. Stem Cells Int 2017: 7617048, 2017.
28. Boumah CE, Selvamurugan $\mathrm{N}$ and Partridge NC: Transcription in the osteoblast: Regulatory mechanisms utilized by parathyroid hormone and transforming growth factor-beta. Prog Nucleic Acid Res Mol Biol 80: 287-321, 2005.

29. Wang Q, Cao L, Liu Y, Zheng A, Wu J, Jiang X and Ji P: Evaluation of synergistic osteogenesis between icariin and BMP2 through a micro/meso hierarchical porous delivery system. Int J Nanomedicine 12: 7721-7735, 2017.

30. Wei Q, Zhang J, Hong G, Chen Z, Deng W, He W and Chen MH: Icariin promotes osteogenic differentiation of rat bone marrow stromal cells by activating the ER $\alpha-W n t / \beta$ catenin signaling pathway. Biomed Pharmacother 84: 931-939, 2016.

31. Mok SK, Chen WF, Lai WP, Leung PC, Wang XL, Yao XS and Wong MS: Icariin protects against bone loss induced by oestrogen deficiency and activates oestrogen receptor-dependent osteoblastic functions in UMR 106 cells. Br J Pharmacol 159: 939-949, 2010.

32. Zhang M, Hu X, Li S, Lu C, Li J, Zong Y, Qi W and Yang H: Hepatoprotective effects of ethyl pyruvate against $\mathrm{CCl} 4$-induced hepatic fibrosis via inhibition of TLR4/NF- $\mathrm{BB}$ signaling and up-regulation of MMPs/TIMPs ratio. Clin Res Hepatol Gastroenterol 42: 72-81, 2018.

33. Zhang L, Wang XZ, Li YS, Zhang L and Hao LR: Icariin ameliorates $\operatorname{IgA}$ nephropathy by inhibition of nuclear factor kappa b/Nlrp3 pathway, FEBS Open Bio 7: 54-63, 2016.

34. Zhang S, Feng P, Mo G, Li D, Li Y, Mo L, Yang Z and Liang D: Icariin influences adipogenic differentiation of stem cells affected by osteoblast-osteoclast co-culture and clinical research adipogenic. Biomed Pharmacother 88: 436-442, 2017.

This work is licensed under a Creative Commons Attribution-NonCommercial-NoDerivatives 4.0 International (CC BY-NC-ND 4.0) License. 